\title{
Ordering and phase diagrams of xenon adsorbed on thin epitaxial $\mathrm{NaCl}(100)$ films and on $\mathrm{Ge}(100)$
}

\author{
C. Schwennicke, J. Schimmelpfennig, and H. Pfnür \\ Institut für Festkörperphysik, Universität Hannover, Appelstraße 2, D-30167 Hannover, Germany
}

(Received 15 April 1993)

\begin{abstract}
An adsorption study of $\mathrm{Xe}$ on both the bare $\mathrm{Ge}(100)$ surface, as well as on thin $\mathrm{NaCl}$ films grown pseudomorphically on $\mathrm{Ge}(100)$, was carried out using high-resolution low-energy electron diffraction. Ordered phases and phase diagrams were investigated in quasiequilibrium with room-temperature gas. Starting from low pressures, a transition from a disordered lattice gas to a distorted hexagonal phase was found for $\mathrm{Xe}$ on $\mathrm{NaCl}(100)$ in addition to the hexagonal bulk phase, which grows as a multilayer phase. The distorted hexagonal phase was found to be commensurate with the substrate in only one direction. At lower temperatures and/or higher pressures, the onset of three-dimensional growth is observed in the form of a hexagonal nanocrystalline phase (average grain size $80 \AA$ ) with Xe bulk lattice constant which is still oriented along the $[110]$ direction with one of its axes. Both phase transitions are of first order, with pronounced hysteresis effects for the second. On $\operatorname{Ge}(100)$, no additional superstructure spots are induced by Xe before the bulk phase grows. The $(2 \times 1)$ reconstruction is maintained, but characteristic jumps in intensity of integral order as well as of half-order beams are observed as a function of temperature and pressure so that Xe seems to lock on defined sites in $(2 \times 1)$ registry. Phase diagrams for Xe adsorbed on both surfaces were derived and heats of transition at the phase boundaries were determined.
\end{abstract}

\section{INTRODUCTION}

Physisorption of noble gases on well-defined surfaces and the investigation of phase transitions between a large manifold of commensurate and incommensurate structures have attracted wide interest in the past. Extensive experimental studies have been carried out mostly on substrates with hexagonal symmetry both for noble gases and weakly bound molecules using diffraction techniques like neutron scattering, ${ }^{1}$ helium scattering, ${ }^{2}$ and electron diffraction, ${ }^{3}$ as well as uptake methods to study anomalies of the specific heat at two-dimensional phase transitions. ${ }^{4}$ What is most interesting in these systems is the interplay of adsorbate-adsorbate interactions and adsorbate-substrate interactions. On a substrate with threefold or sixfold symmetry both adsorbate and substrate potentials have the same symmetry for the case of noble-gas adsorption, but the mismatch between the lattice constants of the substrate and the adsorbate leads to the formation of domain walls with either hexagonal or striped symmetry, or to the formation of floating twodimensional solid phases. ${ }^{5}$

If these symmetries are different, the competition between these adsorbate-adsorbate interactions and the adsorbate-substrate interactions should lead to new ordering phenomena. This is the motivation for our investigation presented here. Attempts in this direction have been made before, e.g., in investigations of Xe on metals like $\mathrm{Cu}(100)$ (Ref. 6) and on $\mathrm{Pd}(100)$ (Ref. 7), and also on the ionic surfaces of $\mathrm{MgO}(100),{ }^{8}$ but only hexagonal structures were found on the metallic surfaces. In this paper we will focus our interest on the ordering of an adsorbed rare gas Xe on surfaces with fourfold and twofold symmetry. As a substrate we have chosen the $\mathrm{NaCl}(100)$ and the $\mathrm{Ge}(100)$ surfaces. Whereas the $\mathrm{NaCl}(100)$ surface remains unreconstructed ${ }^{9,10}$ and thus remains fourfold symmetric also on the surface, the symmetry is further reduced on $\mathrm{Ge}(100)$. Detailed structural investigations ${ }^{11}$ show that the $\mathrm{Ge}(100)$ surface forms a mixture of $(2 \times 1), p(2 \times 2)$, and $c(4 \times 2)$ reconstructions at the temperatures relevant in our study. These structures differ only in position and orientation of adjacent dimer rows relative to each other. Due to these reconstructions the $\mathrm{Ge}(100)$ surface is much more corrugated than the $\mathrm{NaCl}$ surface. As the $\mathrm{NaCl}$ layers can be grown as high-quality thin films on the $\mathrm{Ge}(100)$ surface we had an easy opportunity to switch between surfaces of different adsorbate-substrate coupling and different corrugation, thereby explaining our special choice of substrates.

Ionic crystals with a wide band gap can be studied with low-energy electrons only by using thin films so that charging effects can be avoided to a large extent. Because of the small mismatch in lattice constants of less than $0.5 \% \mathrm{Ge}(100)$ is a favorable substrate for epitaxial growth of $\mathrm{NaCl}^{9}$ The use of such films not only has technical advantages but also makes them accessible to high-resolution structural investigations so that details of the processes of growth and annealing of defects can be studied. ${ }^{12,13}$ In a recent study ${ }^{14,15}$ we showed that $\mathrm{NaCl}$ films can be grown on $\mathrm{Ge}(100)$ as high-quality epitaxial films. The different step heights of $\mathrm{NaCl}$ and $\mathrm{Ge}$ caused by the different crystal structures are accommodated by strains in the $\mathrm{NaCl}$ layers which get smaller but are extended over a larger area with increasing film thickness. The resulting small dilatations (see below) are not expected to have a major influence on the phase transitions studied in this paper. This statement is corroborated comparing infrared (IR) results on phase tran- 
sitions for $\mathrm{CO}_{2}$ on bulk $\mathrm{NaCl}$ (Ref. 16) and corresponding low-energy electron-diffraction (LEED) results on $\mathrm{NaCl}$ films ${ }^{17}$ of similar quality than those used in our study.

In this paper we concentrate on the experimental results of Xe adsorption on the (100) surfaces of $\mathrm{NaCl}$ and Ge. We first describe the LEED patterns on $\mathrm{NaCl}$ and the corresponding real-space structures. The phase transitions between them were determined from characteristic changes in LEED spot intensities and are summarized in a phase diagram. The pressure and temperature dependences of the ordered structures were measured in detail to prove one-dimensional incommensurability of what we call the two-dimensional (2D) phase. A short excursion to ordering kinetics follows. A phase diagram of Xe ordering can also be constructed on $\mathrm{Ge}(100)$ from the characteristic changes of LEED intensities as a function of pressure and temperature, as shown in Sec. IIIE although no Xe-induced superstructures appear. These results will finally be discussed and summarized.

\section{EXPERIMENT}

Experiments were performed in a standard UHV system (base pressure $6 \times 10^{-9} \mathrm{~Pa}$ ) equipped with a highresolution spot-profile analyzing LEED (SPALEED) instrument, an Auger electron spectrometer, and a quadrupole mass spectrometer.

Profile analysis of the diffracted beams was carried out with the SPALEED instrument. As checked by profile measurements at in-phase conditions between different terraces on the clean $\mathrm{Ge}(100)$ substrate, the effective transfer width was found to be larger than $1100 \AA$. This value already contains broadening due to imperfections such as mosaic spread of the crystal. Details of the instrument are described in Ref. 18.

In order to minimize mechanical stress and thermal gradients on the sample the Ge crystals of $10 \times 10 \times 0.6$ $\mathrm{mm}^{3}$ in size were inserted into a molybdenum frame which was attached by small $\mathrm{Ti}$ screws to a Mo sheet. This whole assembly was then mounted to a cryostat which was cooled with liquid He. Temperature was measured on the Mo frame by a Chromel-Alumel thermocouple. The crystal assembly was heated from the back side either by radiation from a filament or by electron bombardment. For measurements only the radiational heating was used. Temperature was regulated by a computerized temperature control to $\pm 0.1 \mathrm{~K}$ and could be ramped linearly up and down. Temperature was calibrated using the onset of bulk Xe formation during condensation at a $\mathrm{Xe}$ pressure of $10^{-4} \mathrm{~Pa}$ on the $\mathrm{NaCl}$ sample, the highest accessible pressure. Hysteresis effects are expected to be the smallest there. Temperature was adjusted so that it was identical to the temperature of the Xe vapor pressure curve at this pressure. The error in absolute temperature is estimated to be $\pm 1 \mathrm{~K}$.

Ge crystals were cut from a massive rod, grinded, etched, and oriented on a x-ray diffractometer to better than $\pm 0.1^{\circ}$. The (100)-oriented Ge disks were then polished mechanically with diamond pastes down to 0.25 $\mu \mathrm{m}$ grain size. Subsequently a chemomechanical polishing step was carried out using a solution of 0.05 vol. \% bromine in methanol. Finally, they were cut to the size mentioned above. After mounting and baking the UHV chamber the surfaces were contaminated by carbon, which was removed in UHV by Ar-ion sputtering at room temperature and subsequent annealing to about $1120 \mathrm{~K}$. This procedure was repeated until no traces of carbon could be identified with Auger spectroscopy even after several cycles of annealing. After preparation the sample showed the well-known $(2 \times 1)$ reconstruction at room temperature and both $c(4 \times 2)$ and $p(2 \times 2)$ reconstructed regions at lower temperatures. ${ }^{19}$

In order to determine the step density on the clean $\operatorname{Ge}(100)$ surface we measured and fitted profiles of the (00) spot as a function of beam energy. The half width of the (00) spot in the out-of-phase condition with respect to steps of monoatomic height $d=1.4 \AA$ was $1.0 \%$ of the diameter of the first Brillouin zone (BZ) in the $\overline{\Gamma X}$ direction after deconvolution with the instrumental response function measured at the in-phase condition. Hence, the mean separation of monoatomic steps was about $250 \AA$ on the clean $\mathrm{Ge}(100)$ surface.

$\mathrm{NaCl}$ was evaporated onto the substrate at a substrate temperature of $200 \mathrm{~K}$. The deposition rate was monitored by a quartz microbalance. We calibrated the microbalance by measuring the $\mathrm{Cl}(L M M) 181 \mathrm{eV}$ Auger signal as a function of the frequency shift of the quartz up to the first kink with an uncertainty and reproducibility of $\pm 10 \%$. The calibration procedure is described in detail elsewhere. ${ }^{9}$ The film thickness used in all our measurements described below was $17 \AA$, i.e., three double layers. Between adsorption measurements the sample was routinely heated to $650 \mathrm{~K}$ in order to remove any traces of impurities adsorbed from the background pressure.

The morphology of the epitaxial $\mathrm{NaCl}$ film is described and discussed in detail elsewhere. ${ }^{14} \mathrm{We}$ found that the step density on the epitaxial $\mathrm{NaCl}(100)$ film is not larger than on the $\mathrm{Ge}(100)$ surface, i.e., the average terrace length of the $\mathrm{NaCl}$ film was also found to be approximately $250 \AA$. Note, however, that steps of monoatomic height of the germanium diamond lattice cannot exist in the $\mathrm{NaCl}$ lattice. Since an accommodation of these steps by the $\mathrm{NaCl}$ film on the scale of one lattice constant would produce large Coulomb fields, the $\mathrm{NaCl}$ film accommodates these steps gradually, i.e., over several lattice constants by an elastic distortion. A quantitative analysis of the LEED spots using a film thickness of three double layers, described in Ref. 14, shows that this accommodation of a step of the Ge surface is distributed over about 20 lattice constants $(80 \AA)$, i.e., onethird of the mean separation of monoatomic steps. If these slightly distorted areas are assumed to be flat and uniformly strained, tilt angles of less than $1^{\circ}$ and local changes of the lattice constant of $2.2 \%$ are obtained. As these changes are so small, we do not expect that they have an important influence on the properties of adsorbed layers.

Xenon was admitted to the vacuum chamber through a variable leak valve. Adsorption experiments were performed in quasiequilibrium with a three-dimensional (3D) room-temperature gas. At the smallest Xe pressures used, the Xe gas in the chamber contained about $3 \%$ of molecular hydrogen, the main constituent of the resid- 
ual gas in the chamber. Since $\mathrm{H}_{2}$ does not physisorb at temperatures above $30 \mathrm{~K}$, we do not expect any influence on our results. Pressures of all other constituents of the residual gas were at least one order of magnitude smaller than that of $\mathrm{H}_{2}$. Xe pressure was measured using an ion gauge. Pressures were corrected by a factor 0.33 taking into account the larger ionization cross section of xenon with respect to $\mathrm{N}_{2}$. The LEED patterns were recorded as a function of temperature at constant pressure, and also as a function of time at constant temperature and pressure.

\section{RESULTS}

\section{A. Superstructures of $\mathrm{Xe}$ adsorbed on $\mathrm{NaCl}$}

Two different Xe-induced superstructures were observed on $\mathrm{NaCl}$. Decreasing the temperature below 60 $\mathrm{K}$ at a constant xenon pressure above $3 \times 10^{-5} \mathrm{~Pa}$ the LEED pattern shown in Fig. 1(a) appears first. Characteristic are pairs of superstructure spots which are, for the lowest orders of diffraction, positioned exactly in the middle between $\mathrm{NaCl}$ substrate spots. The pairs have equal intensities only for the innermost pairs, but differ greatly in intensity for higher orders. We note that, independent of electron energy, the eight spots marked by squares in Fig. 1(b) always appear with approximately equal intensities relative to each other, and are generally much brighter than the other superstructure spots. As an example, the one-dimensional scan shown in Fig. 2, starting close to the $(1 / 2,0)$ position along the $[01 \overline{1}]$ direction, shows a dominant superstructure spot at the right [marked by squares in Fig. 1(b)], and three additional superstructure spots with much less intensity. From this observation we tentatively consider these bright spots as first-order spots of the superstructure. From symmetry considerations the first-order substrate spots also must belong to this superstructure. This superstructure has to exist in two domains, because the square symmetry is broken (see Fig. 1).

According to these observations we identify the spots marked by squares as single diffraction spots from the adsorbate structure, and obtain the unit cells of the Xe superstructure as shown on Fig. 1(b). The additional spots are due to multiple scattering from both the substrate and the adsorbate and can be generated by the addition of the wave vectors from substrate and adsorbate spots. This picture is consistent up to the highest diffraction orders shown in Fig. 1.

A real-space representation of the Xe superstructure is shown in Fig. 3, which can be obtained after Fourier transformation of the LEED pattern assuming the spots marked by squares as first-order spots. The primitive vectors $\mathbf{a}_{1}^{\text {ads }}$ and $\mathbf{a}_{2}^{\text {ads }}$ generate the unit mesh of the adsorbate structure of domain $B$. Since the (01) spots from the substrate and adsorbate coincide, the $y$ components of $\mathbf{a}_{2}^{\text {ads }}$ and $\mathbf{a}_{2}^{\text {subs }}$ must be equal. Thus the distance of two adjacent xenon rows is identical to the lattice constant $a_{0}=3.96 \AA$ of the $\mathrm{NaCl}(100)$ surface. On the other hand, the distance of two adjacent xenon atoms within a row can be determined from the distance $\Delta K$ of two innermost adjacent multiple-scattering spots using

$$
d_{\mathrm{Xe}-\mathrm{Xe}}=\frac{a_{0}}{1-\left(a_{0} \Delta K / 4 \pi\right)} .
$$

The distance of the multiple-scattering spots depends on pressure and temperature, as dicussed later, but is al-

(a)

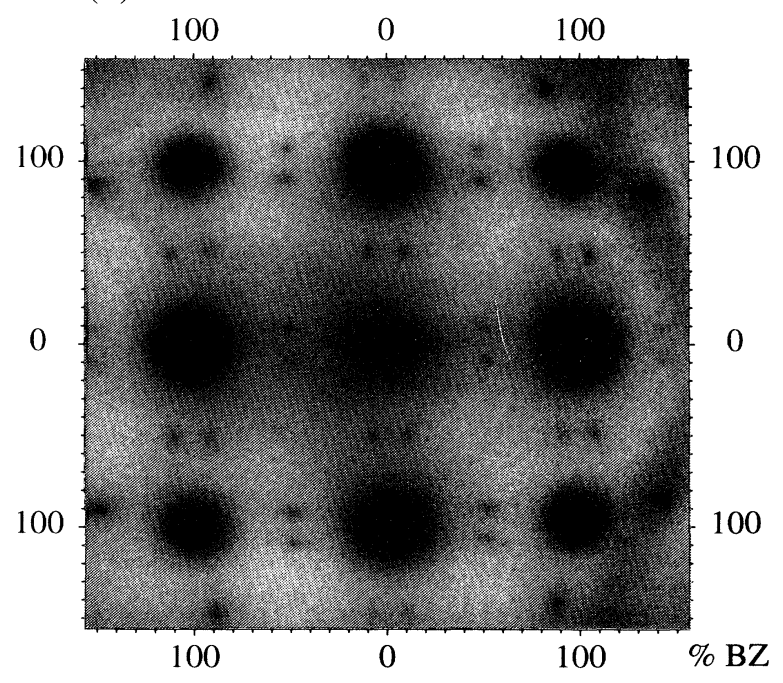

(b)

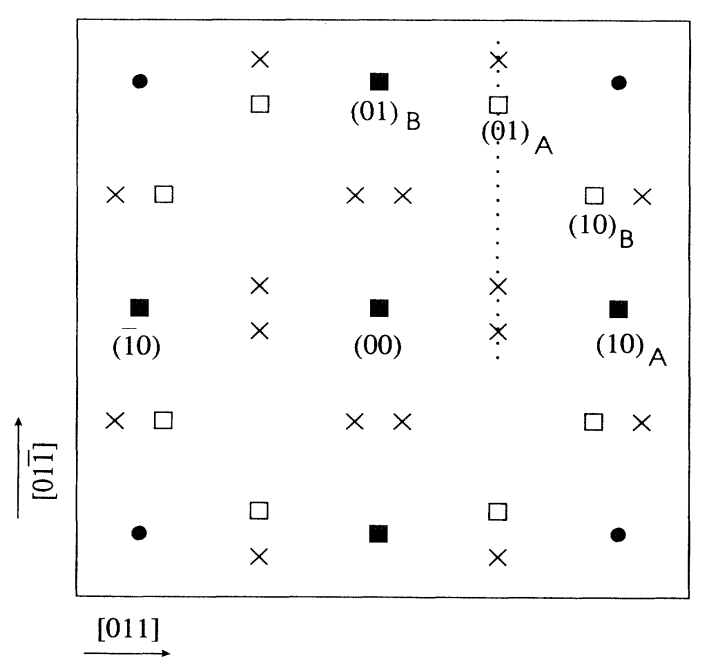

FIG. 1. (a) LEED pattern of $\mathrm{Xe}$ adsorbed on $\mathrm{NaCl}$ at $T=50 \mathrm{~K}, p=1 \times 10^{-5} \mathrm{~Pa}$, and $E=82 \mathrm{eV}$ showing the (00) spot in the middle. Dark spots are the $\mathrm{NaCl}$ spots. Their width is due to overexposure and is not real. Distances between diffraction spots are measured in units of the separation between (00) and (10) spots of $\mathrm{NaCl}$, corresponding to the diameter of the first Brillouin zone (BZ). (b) Schematic of the LEED pattern shown in (a). Open squares, single diffraction adsorbate spots; filled squares, spots common to adsorbate and substrate; filled circles, substrate spots; crosses, multiplescattering spots; dots, path of scan in Fig. 2. 


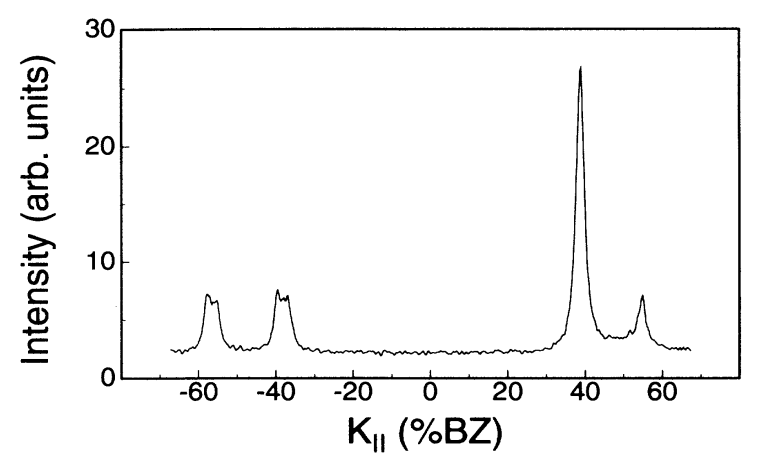

FIG. 2. One-dimensional scan through the LEED pattern shown in Fig. 1(a) along the dotted line in Fig. 1(b). Note that the large half widths of the left two multiple-scattering spots are not real but due to instrumental defocusing. The instrument was optimized for the spots at approximately $40 \%$ and $60 \% \mathrm{BZ}$.

most constant below $53 \mathrm{~K}$. At these temperatures we determined the distance $d$ between two adjacent Xe atoms within a row as $4.37 \AA$, in excellent agreement with the well-known bulk lattice constant in this temperature range. ${ }^{20}$ Hence, the distance between adjacent Xe atoms is determined in one direction by the Xe bulk lattice constant and does not lock into the $\mathrm{NaCl}$ substrate periodicity along this direction, whereas in the other direction the Xe layer is expanded by $5.5 \%$ so that it fits on the $\mathrm{NaCl}$ substrate and is commensurate in this direction. This results in a distorted hexagonal structure of the $\mathrm{Xe}$ overlayer with an angle between primitive Xe vectors of $118.7^{\circ}$ instead of $120^{\circ}$ in the bulk.

The perfectness of the Xe layers was found to be strongly dependent on substrate imperfections of $\mathrm{NaCl}$ and/or Ge, as seen from the half widths of Xe superstructure spots. In all cases, two-dimensional high-resolution spot profiles of Xe superstructure spots showed an elliptical intensity distribution. For the most perfect conditions obtainable, the full width at half maximum of the $(01)_{A}$ spot was about $1.6 \% \mathrm{BZ}$ along the [011] and $1 \% \mathrm{BZ}$ along the $[01 \overline{1}]$ direction. This shows that under these conditions long-range order in the Xe layers is only limited by

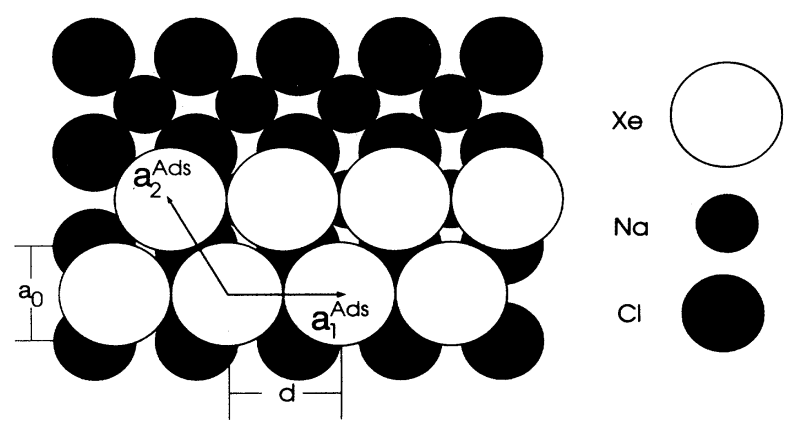

FIG. 3. Real-space representation of the structure model of the Xe monolayer adsorbed on $\mathrm{NaCl}$. Only one domain is shown.

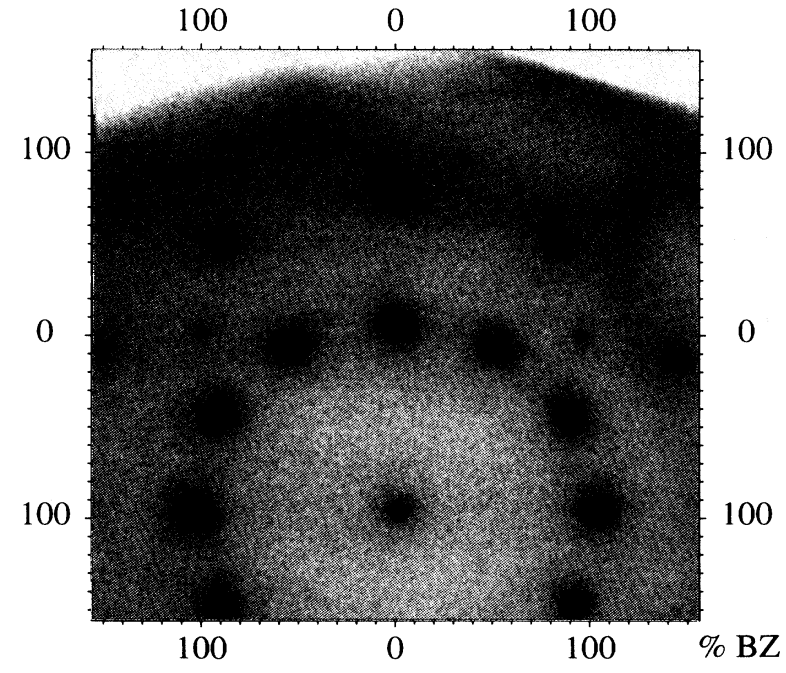

FIG. 4. LEED pattern of the 3D bulk structure of $\mathrm{Xe}$ condensed on $\mathrm{NaCl}$. Note that the $(00)$ spot is at position $(0$, $-90)$.

the mean terrace length of the $\mathrm{NaCl}$ film along one direction. The limited order in the direction where the $\mathrm{Xe}$ superstructure is in registry to the substrate, i.e., perpendicular to the close-packed Xe rows, means that the order of the superstructure in this direction is disturbed by moving complete Xe rows of one domain against each other. Obviously, the periodicity of the adsorbate structure is less disturbed along the close-packed rows from which the observed asymmetry results.

After further decreasing the temperature a second LEED pattern appears which is shown in Fig. 4. The twelvefold symmetry of the LEED pattern can easily be shown to be due to two orthogonal hexagonal domains. The exact orientation with respect to the $\mathrm{NaCl}$ substrate and the lattice constants of this phase were measured on thin layers where both the $\mathrm{NaCl}$ and the Xe diffraction spots are still visible (see Fig. 4). We found that one crystallographic direction of these Xe layers is still oriented along the [011] direction of the substrate. From the splitting between (10) $\mathrm{NaCl}$ and (10) xenon spots we calculate the lattice constant of this hexagonal Xe phase as $4.37 \AA$, which again agrees with the value of the bulk lattice constant. ${ }^{20}$

As the formation of this phase is combined with strong damping of the $\mathrm{NaCl}$ diffraction spots, and their gradual disappearance as a function of time once the critical pressure is exceeded, it is most likely that this bulk phase is only formed in the second Xe layer. The orientation of thicker Xe layers is the same as for the second layer described above. From the half width of the hexagonal spots we get a mean lateral size of the 3D xenon crystallites of about $80 \AA$.

\section{B. Phase diagram}

We have investigated xenon adsorption on $\mathrm{NaCl}$ at constant xenon partial pressures between $3 \times 10^{-6} \mathrm{~Pa}$ 
and $1 \times 10^{-4} \mathrm{~Pa}$. Before each isobaric measurement the sample was heated to $650 \mathrm{~K}$ in order to remove residual impurities. Measurements were routinely started at a temperature of $70 \mathrm{~K}$. After reaching this temperature, xenon was admitted to the vacuum chamber. Temperature was then lowered in steps of $0.1 \mathrm{~K}$ and profiles of both the (10) substrate spot and the $(01)_{A}$ superstructure spot were recorded at constant temperature. If the intensity of one spot changed in two consecutive measurements the temperature was kept constant and spot profiles were recorded again.

In Fig. 5 integrated intensities of the $\mathrm{Xe}(01)_{A}$ spot are plotted as a function of temperature. They all show a stepwise change of intensities at characteristic temperatures, indicating the phase transition from a twodimensional gas phase to the ordered distorted hexagonal phase of the first Xe layer. Deviations from step functions within our resolution can be seen for the lowest pressure measured. It seems that adsorption and/or diffusion kinetics had become so slow that a full equilibrium was not reached. In order to check the reversibility of the isobars the superstructure spot intensity was recorded in adsorption and desorption at pressures $p=3.3 \times 10^{-5}$ $\mathrm{Pa}$ and $p=1.3 \times 10^{-5} \mathrm{~Pa}$. Within the limit of experimental resolution no hysteresis could be observed. The isobars were fully reversible if growth of the hexagonal bulk phase was avoided. The points of half-maximum intensity were taken as the points of the phase transitions. These were plotted in Fig. 6.

Contrary to the adsorption of the monolayer the bulk condensation shows considerable hysteresis effects in temperature sweeps up and down. Temperature sweeps were carried out at $-0.1 \mathrm{~K} / \mathrm{min}$ for sweeps downward, but a factor of 4 faster in the upward direction. Therefore, the data obtained during lowering the temperature are closer to equilibrium, and are shown in Fig. 6 for three different Xe partial pressures. For the lowest pressure $\left(2 \times 10^{-5} \mathrm{~Pa}\right)$ a hysteresis of $5 \mathrm{~K}$ was observed. It is very obvious that the uncertainty in the determination of the $2 \mathrm{D}$ to $3 \mathrm{D}$ transition points is much higher than of the gas-2D transition points. The heats of transition were calculated for the gas-2D and the 2D-3D phase transitions using the Clausius Clapeyron equation. We

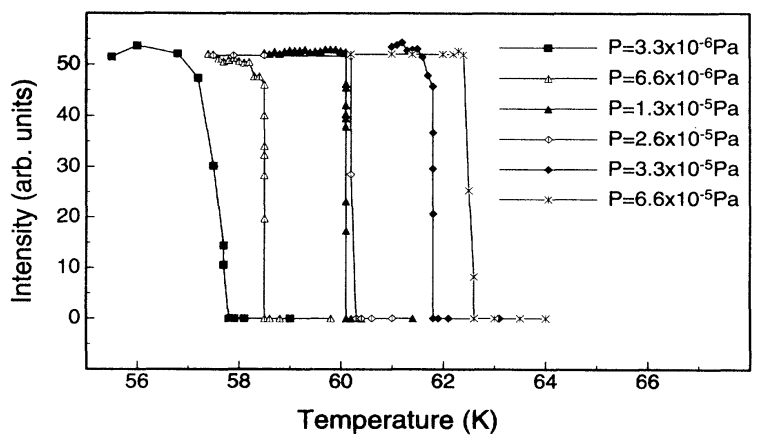

FIG. 5. Integrated superstructure LEED spot intensities as a function of temperature for several Xe partial pressures (isobars).

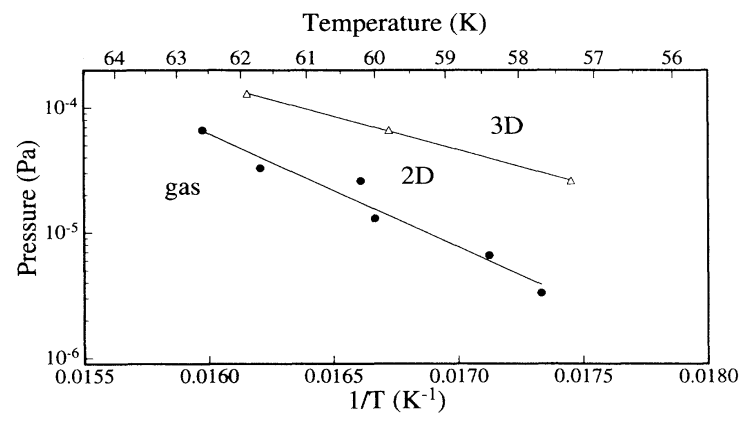

FIG. 6. Phase diagram of xenon adsorbed on $\mathrm{NaCl}$.

obtain a value of $17.3 \mathrm{~kJ} / \mathrm{mol}$ for the transition from a $2 \mathrm{D}$ gas to a $2 \mathrm{D}$ solid, and a value of $10.3 \mathrm{~kJ} / \mathrm{mol}$ for the $2 \mathrm{D}-3 \mathrm{D}$ phase transition. The value obtained for the phase transition to a two-dimensional solid compares well with results obtained by Hardy et al. ${ }^{21}$ with photoelectron spectroscopy and with results from thermal programmed desorption (TPD). ${ }^{10}$ Our values are 5-10\% smaller than those obtained by the other methods. Taking into account the experimental uncertainties these differences cannot be considered to be significant. The value for the 2D-3D transition, on the other hand, is considerably smaller than the condensation enthalpy of a Xe solid [15.5 kJ/mol (Ref. 22)]. The discrepancy clearly stems from the nonequilibrium effects mentioned. The corresponding line in the phase diagram should only be taken as a rough indication of this phase transition, therefore.

\section{Temperature and pressure dependence of the lattice constant}

From a single LEED pattern we cannot distinguish between an incommensurate and a high-order commensurate adsorbate structure. However, an unambiguous identification of an incommensurate structure is possible considering the variation of the lattice constant with respect to temperature, if the thermal expansion of the substrate is negligible in a small temperature range. A very precise determination of the $\mathrm{Xe}-\mathrm{Xe}$ spacing $d_{\mathrm{Xe}-\mathrm{Xe}}$ with an uncertainty of about $0.005 \AA$ along the incommensurate direction is possible by measuring the distance $\Delta K$ between adjacent multiple-scattering spots according to Eq. (1). Figure 7 shows the variation of $d_{\mathrm{Xe}-\mathrm{Xe}}$ as a function of temperature for the hexagonal distorted phase. These data were obtained in two different ways.

(i) Below $48 \mathrm{~K}$ the equilibrium pressure was too small, so the gas was pumped away after adsorption. In this temperature range the desorption rate of $\mathrm{Xe}$ is negligible, and we measured at an appropriate constant coverage.

(ii) Above $48 \mathrm{~K}$ this ordered phase was produced by adjusting the xenon pressure so that the measurements were performed in the middle between the phase boundaries. After reaching equilibrium conditions the distance of the multiple-scattering spots was measured.

Below $55 \mathrm{~K}$ the measured xenon-xenon spacing was found to be approximately equal to the bulk lattice constant. Above $55 \mathrm{~K}$ a drastic increase of $d_{\mathrm{Xe}-\mathrm{Xe}}$ with in- 


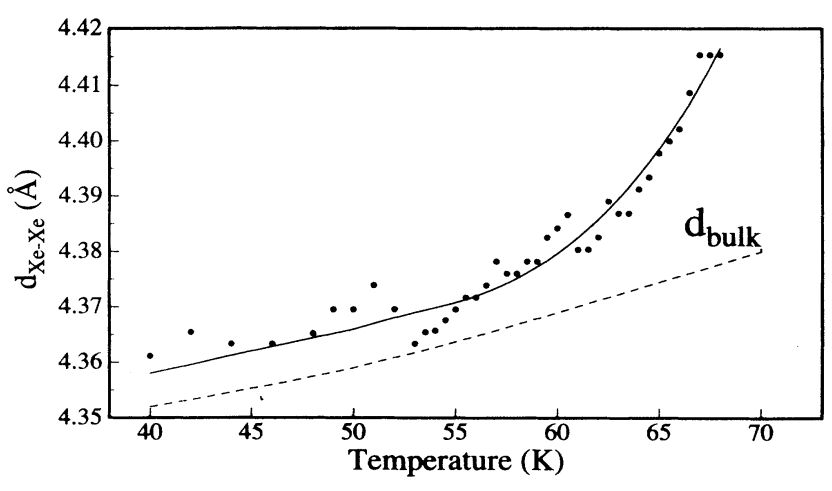

FIG. 7. Xe-Xe spacing along the incommensurate direction as a function of temperature.

creasing temperature was found. The one-dimensional expansion coefficient is strongly increasing above $55 \mathrm{~K}$ and exceeds by far the value for bulk Xe at these temperatures. Note that the expansion of the overlayer is uniaxial since the xenon monolayer is in registry to the substrate along one direction.

We have also determined $d_{\mathrm{Xe}-\mathrm{Xe}}$ as a function of the xenon partial pressure at constant temperature $T=60$ $\mathrm{K}$ in order to investigate the isothermal compression of the overlayer. Results are shown in Fig. 8. A decrease is seen of $d_{\mathrm{Xe}-\mathrm{Xe}}$ from $4.40 \AA$ at the gas-2D phase boundary to $4.38 \AA$ at the $2 \mathrm{D}-3 \mathrm{D}$ phase boundary. Since strong changes of the lattice constant could be observed only close to the gas-2D phase boundary the variation of the lattice constant as a function of temperature is not strongly influenced by the pressure dependence as these measurements were performed in the middle of the $2 \mathrm{D}$ region. The scatter in the data above $55 \mathrm{~K}$ of Fig. 7 reflects these variations at constant temperature again. It is in the limit of experimental accuracy of $\pm 0.005 \AA$.

\section{Ordering kinetics}

Time-dependent ordering was measured using spot profiles and integrated LEED intensities of superstruc-

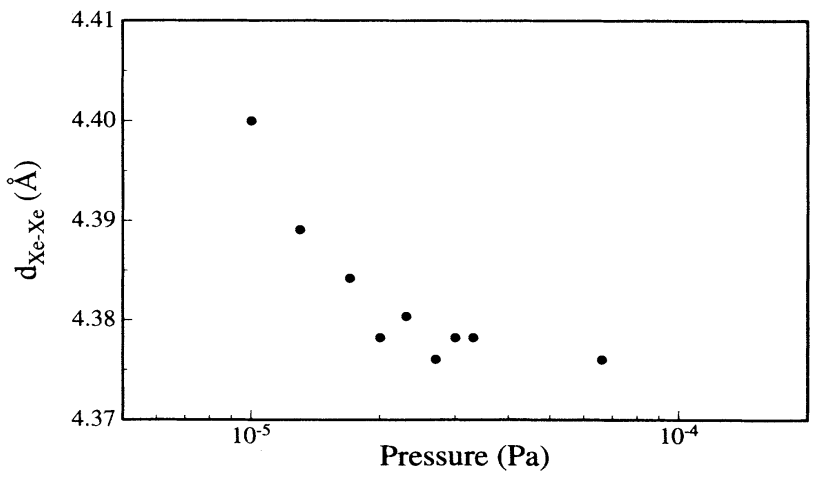

FIG. 8. Xe-Xe spacing along the incommensurate direction as a function of pressure at constant temperature $T=60$ $\mathrm{K}$. ture spots at constant pressure and temperature. Before each measurement the sample was heated to $650 \mathrm{~K}$ in order to remove residual adsorbates. After reaching a constant temperature $T=54 \mathrm{~K}$ gas was admitted to the vacuum chamber at the initial time $t=0$ and the corresponding xenon partial pressure was adjusted. As the phase diagram shows, xenon pressures between $5 \times 10^{-7}$ $\mathrm{Pa}$ and $5 \times 10^{-6} \mathrm{~Pa}$ correspond to the $2 \mathrm{D}$ phase at a temperature of $54 \mathrm{~K}$.

First, spot profiles of a superstructure spot were recorded during adsorption and desorption. The shape of these profiles turned out to be remarkably constant once intensity exceeded $15 \%$ of the saturation intensity. A typical example, not for the lowest defect concentrations of the substrate, is shown in Fig. 9 during adsorption at $P=5 \times 10^{-7} \mathrm{~Pa}$. This result was independent of the defect density of the substrate. The absence of spot profile changes as a function of increasing coverage cannot be easily understood in the context of simple island formation during growth, because a constant average island size and a varying number of islands would have to be assumed. In addition, it seems that defects induce stacking faults, i.e., domain formation, in the various directions. This causes broadening of the profiles. Assuming that the average domain size is only dependent on the defect density, the domain size would not vary as a function of coverage, in agreement with our measurements. Although no systematic study has been carried out, we interpret this result as indicative of island formation with an average size of undistorted domains determined by the average distance of surface defects, starting at a coverage as low as $15 \%$ of the saturation coverage.

Figure 10 shows the integrated intensities of the superstructure spots at $T=54 \mathrm{~K}$ and at three different xenon pressures as a function of time. Spot profiles were integrated in two dimensions and the background intensity, measured in the middle between (00) and (11) spot, was subtracted from the intensities. The smallest value of the xenon pressure $P=5 \times 10^{-7} \mathrm{~Pa}$ corresponds to a point close to the gas- $2 \mathrm{D}$ phase boundary. Two fea-

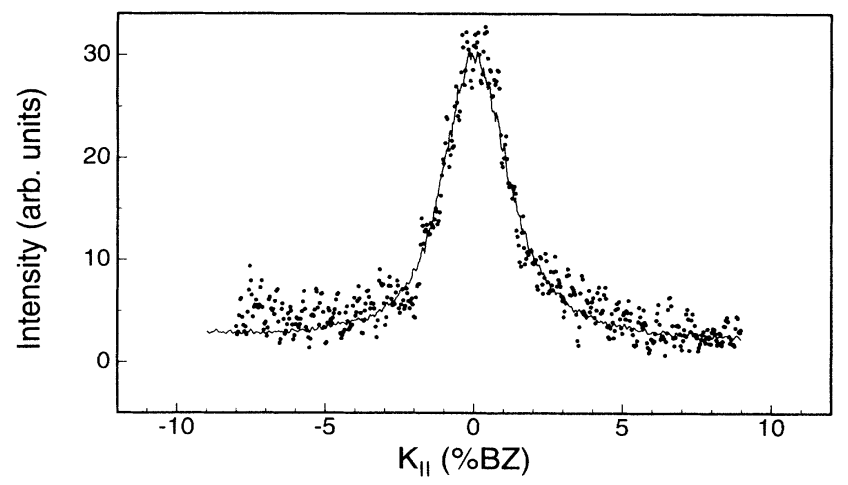

FIG. 9. Normalized spot profiles of the $(01)_{A}$ superstructure spot during adsorption at $T=54 \mathrm{~K}$ and $P=5 \times 10^{-7}$ $\mathrm{Pa}$ at different coverages showing constant profile shapes as a function of coverage. Solid line, saturation coverage; dots, $15 \%$ of the saturation coverage. 


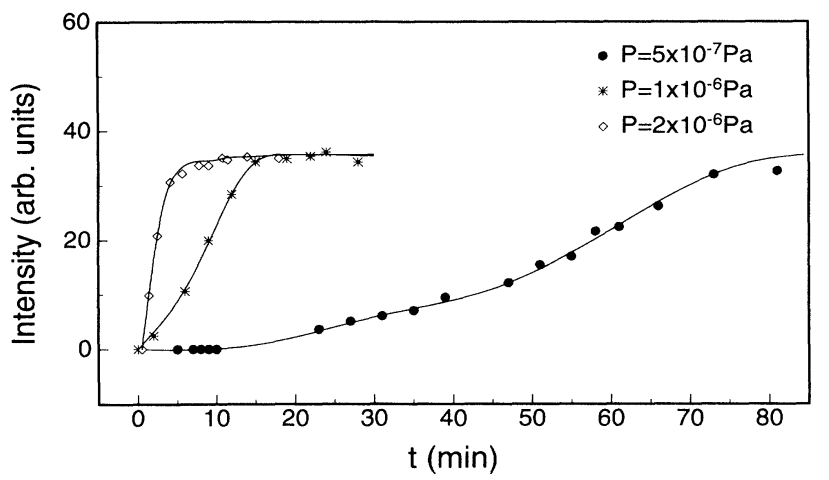

FIG. 10. Integrated intensities of the $(01)_{A}$ superstructure spot at $T=54 \mathrm{~K}$ and different values of the pressure $P$. At the initial time $t=0$ gas was admitted to the chamber and the pressure was adjusted.

tures in these curves should be noted. First, there is a delayed onset of measurable intensity. This can be easily understood from the phase diagram shown in Fig. 6. A low-density lattice-gas phase must exist at low coverages before the $2 \mathrm{D}$ ordered phase is formed. During adsorption at a constant adsorption rate and at a temperature so that formation of the ordered $2 \mathrm{D}$ phase is possible, this phase is only formed after exceeding a critical coverage causing the delayed onset of superstructure beam intensity. Second, the slope of these intensities as a function of time increases. The integrated intensities are directly proportional to the amount adsorbed as an ordered layer. This can be set equal to the total amount adsorbed if one neglects the coverage adsorbed as a dilute lattice gas. The increasing slope then indicates an increasing sticking coefficient as a function of coverage. With the latter assumption we can estimate the maximum sticking coefficient from the steepest slope of the integrated LEED intensities (see Fig. 10), which appears close to saturation of the intensities. In order to avoid influences of redesorption, differences of uptake curves close to saturation at different pressures but constant temperature, were calculated. Assuming that the rate of desorption only depends on temperature in the small coverage range considered here, it can be subtracted out

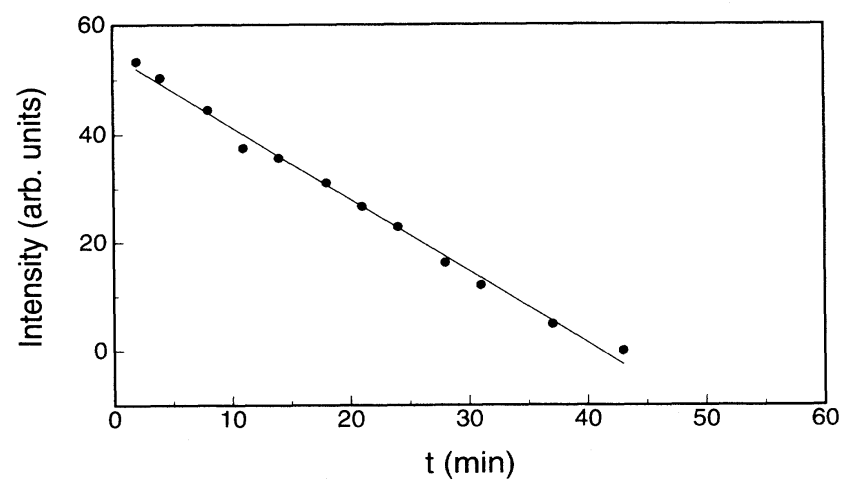

FIG. 11. Integrated intensity of the $(01)_{A}$ superstructure spot at $T=54 \mathrm{~K}$ during desorption $\left(P_{\mathrm{Xe}}<1 \times 10^{-7} \mathrm{~Pa}\right)$. in this way. From these slopes we calculate a maximum sticking coefficient of $S=0.95 \pm 0.08$ for $T=54 \mathrm{~K}$, which is not significantly different from unity. The sticking coefficient at the onset of formation of the ordered phase is smaller by at least a factor of 3 .

In desorption, on the other hand, a linear decrease of the superstructure beam intensity was found, as shown in Fig. 11, which is compatible with a desorption rate independent of coverage as long as we can assume that the density of the condensed islands is much larger than the density of the disordered phase.

\section{E. Xe adsorption on $\mathrm{Ge}(\mathbf{1 0 0})$}

It is of special interest to compare the results obtained on the $\mathrm{NaCl}(100)$ surface with those on the bare Ge substrate since the lattice constants of both substrates are identical but the fourfold point symmetry of the unreconstructed surfaces is reduced to a twofold symmetry on Ge due to the predominant $(2 \times 1)$ reconstruction ${ }^{11}$ which also makes the corrugation of the Ge surface much larger. Xenon adsorption on the $\mathrm{Ge}(100)$ surface was studied at temperatures between 40 and $80 \mathrm{~K}$ and at xenon pressures between $10^{-6}$ and $2 \times 10^{-4} \mathrm{~Pa}$. In the whole pressure and temperature range no additional superstructure spots due to xenon adsorption could be observed before $3 \mathrm{D}$ condensation started. With $\mathrm{Xe}$ adsorbed, all reconstructions of the clean Ge surface could still be observed. Peak intensities of the $(10)$ and $(1 / 2,0)$ spot were recorded as a function of temperature for constant pressure. Figure 12(a) shows typical data for the $(1 / 2,0)$ beam at a pressure of $2 \times 10^{-5} \mathrm{~Pa}$. The temperature was linearly ramped down with a rate of $0.05 \mathrm{~K} / \mathrm{s}$. Above about $77 \mathrm{~K}$ no changes in intensities could be measured, whereas below $77 \mathrm{~K}$ we observed two jumps of the intensity and a plateau between them. Stepwise changes in intensity were also observed for other diffracted beams at the same temperatures and pressures. Both steps occurred before bulk xenon started to grow. This phase can easily be discriminated as it forms the well-known hexagonal LEED pattern described above. This does not happen, however, for small cooling rates $(0.05 \mathrm{~K} / \mathrm{s})$. Under this condition only an amorphous layer is formed at temperatures below $56 \mathrm{~K}$. The hexagonal pattern was only observed if the sample was cooled fast $(1 \mathrm{~K} / \mathrm{s})$ from 77 $K$, or if adsorption was started on the clean sample at temperatures below $50 \mathrm{~K}$.

In Fig. 12(b) measurements were performed both in adsorption and desorption in order to test the reversibility of the adsorption isobars. Only the first jump in spot intensity is reversible, whereas a hysteresis was observed for the second one.

From a set of data as shown in Fig. 12 (measurements during adsorption) the heats of transition were determined from plots of $\ln P$ versus $1 / T$ as shown in Fig. 13 . We obtain values of $22.5 \pm 0.8 \mathrm{~kJ} / \mathrm{mol}$ for the left curve, and $16.9 \pm 0.8$ for the right curve. The onset of threedimensional growth as an amorphous layer could not be determined since the decrease of the spot intensity of the substrate spots was too slow during the bulk condensation, and no well-defined threshold could be detected. A 

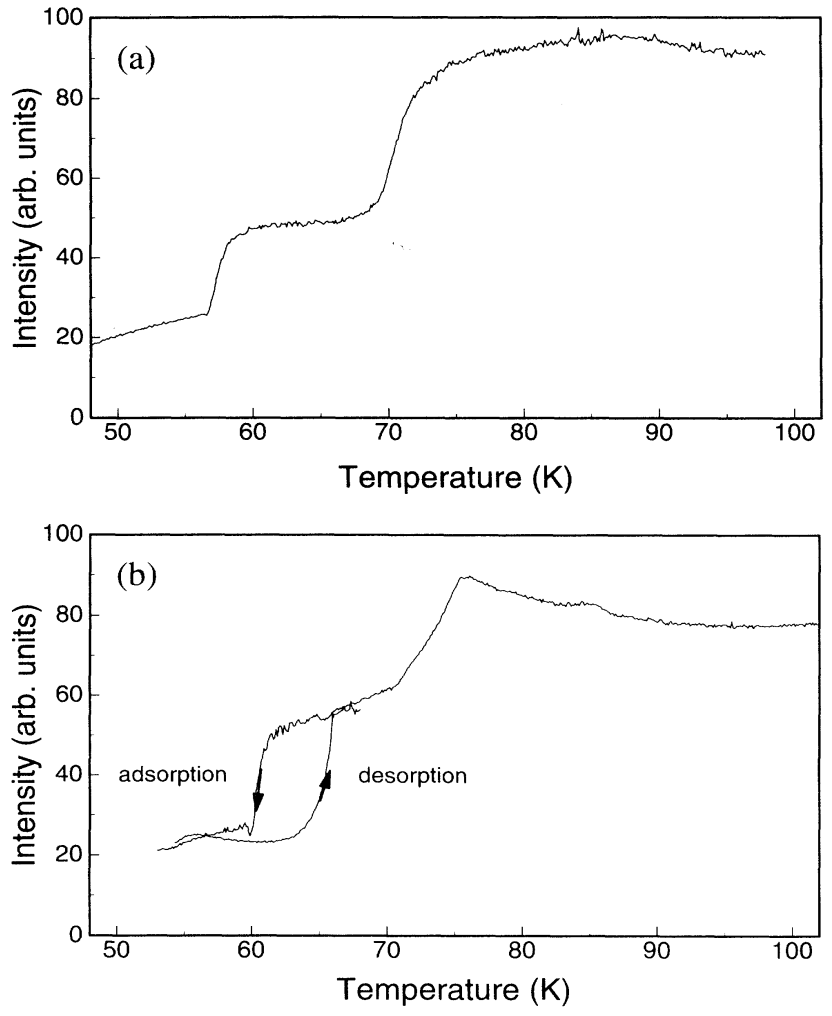

FIG. 12. (a) Peak intensity of the $(1 / 2,0)(a)$ and the (10) spot (b) as a function of temperature at constant pressures $P=2 \times 10^{-5} \mathrm{~Pa}$ and $P=1.3 \times 10^{-4} \mathrm{~Pa}$, respectively, showing hysteresis effects between temperature sweeps up and down in (b).

very similar behavior was found during $\mathrm{Xe}$ adsorption on the $\mathrm{Ge}(111)$ surface. ${ }^{23}$ It seems that the formation of the hexagonal crystalline bulk structure is prevented by the adsorption of ordered $\mathrm{Xe}$ in the first layer. These layers must have the symmetry of the substrate, since no additional spots or features could be observed in the LEED pattern. This difficulty of three-dimensional island growth with the bulk Xe structure may not be surprising since the twofold symmetry of the substrate and the first Xe layer supplies a very poor template for layerby-layer growth.

\section{DISCUSSION}

Our results show that a well-ordered Xe monolayer is formed on the $\mathrm{NaCl}(100)$ surface. Due to the interaction with the fourfold substrate an interesting kind of symmetry frustration phenomenon could be observed. On a smooth substrate the Xe overlayer would order as a close-packed hexagonal structure due to van der Waals interactions between adsorbate atoms. This was found on smooth metal surfaces like $\mathrm{Cu}(100)$ (Ref. 6) or $\mathrm{Pd}(100) .^{7}$ $\mathrm{Xe}$ forms incommensurate hexagonal structures on these surfaces so that the fourfold substrate potential does not determine the adsorbate structure. The $\mathrm{Xe} / \mathrm{NaCl}$ case studied here seems to be a limiting case where the corrugation of the substrate potential and the lateral interac-

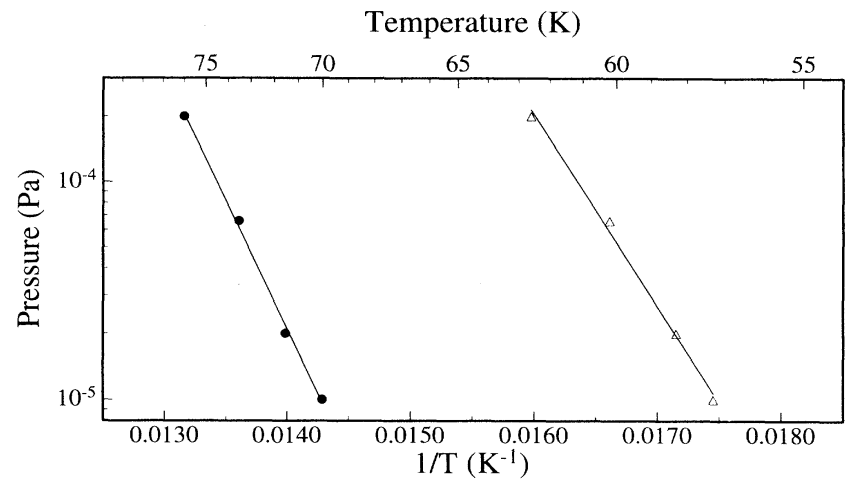

FIG. 13. Plots of $\ln P$ versus $1 / T$ for the first and second jump of the spot intensity which can uniquely be identified in the measured pressure and temperature range.

tions are of the same order of magnitude. This leads to a distortion of the hexagonal adsorbate layer and to onedimensional commensurability. A very similar behavior was observed for $\mathrm{Ar} / \mathrm{MgO}(100)$ (Ref. 8) where the mismatch of lattice constants between bulk $\mathrm{Ar}$ and $\mathrm{MgO}$ is about as large as for $\mathrm{Xe} / \mathrm{NaCl}(100)$. From LEED measurements and potential calculations these authors showed that Ar atoms adsorb close to the $\mathrm{Mg}^{+}$ions forming commensurate rows, i.e., the distance between adjacent adsorbate atom rows is again equal to the substrate lattice constant as in the case of $\mathrm{Xe} / \mathrm{NaCl}(100)$. Parallel to the adsorbate atom rows, however, they found a uniaxial change of lattice constant as a function of gas pressure, which is one order of magnitude larger than in our case. Whereas the observable changes in the lattice constant in the case of $\mathrm{Xe} / \mathrm{NaCl}(100)$ were in the range of only $1 \%$, these changes are around $20 \%$ for $\mathrm{Ar} / \mathrm{MgO}(100) .{ }^{8}$ These large differences in the stability of one-dimensionally commensurate phases remain unclear at the moment. In this context especially the influence of defects, which could stabilize ordered islands, needs to be discussed. Although defect-induced influences on long-range order were still detectable for $\mathrm{Xe} / \mathrm{NaCl}(100)$ we take the stepwise shape of the isobars as an indication for high quality and homogeneity of the epitaxial $\mathrm{NaCl}$ films since it is well known that a high defect density on a substrate surface leads to a smearing out of isobars at a first-order phase transition. A sharp first-order phase transition from a disordered gas to a $2 \mathrm{D}$ phase was also found for $\mathrm{CO}_{2}$ adsorbed on epitaxial $\mathrm{NaCl}$ films. ${ }^{17} \mathrm{On}$ the other hand, we know that our films are strained by about $2 \%$ in the inclined areas close to step edges of the Ge substrate. ${ }^{14}$ These small changes in the lattice constant obviously do not influence the adsorption of $\mathrm{Xe}$ on epitaxial $\mathrm{NaCl}$ films. No differences between results on $\mathrm{NaCl}$ cleavage surfaces and those on thin films prepared in a similar way as ours were also found for the system $\mathrm{CO}_{2} / \mathrm{NaCl}(100)$, as shown recently. ${ }^{16,17}$

The formation of a well-ordered $2 \mathrm{D}$ phase found here seems to disagree with very recent results from Klekamp and Umbach, ${ }^{10}$ where a completely different growth mode of the Xe layer was found. From their TPD, xray photoemission spectroscopy, ultraviolet photoemis- 
sion spectroscopy, and x-ray Auger electron spectroscopy investigations they concluded that $\mathrm{Xe}$ grows as threedimensional islands on the $\mathrm{NaCl}$ film without wetting the surface (Vollmer-Weber growth). Their experimental conditions, however, were quite different from ours. In this study adsorption was carried out irreversibly at around $40 \mathrm{~K}$ in contrast to our equilibrium measurements with a xenon background pressure at substrate temperatures of about $60 \mathrm{~K}$. Nevertheless, it seems doubtful that a change in adsorption temperature can produce this drastic change of growth mode. According to our opinion these discrepancies demonstrate the urgent need for more precise characterization of the surfaces of ionic crystals. In this respect we are still at the beginning.

Our results during irreversible adsorption indicate that the sticking coefficient of xenon is increasing with increasing coverage in the coverage range investigated, i.e., it is smaller on an uncovered surface than on a Xe-covered surface. Accommodation of impinging Xe atoms, i.e., the energy transfer of kinetic energy to the surface, is obviously more efficient on Xe already adsorbed than on the bare $\mathrm{NaCl}$ surface. This behavior is, at first glance, similar to the one found for adsorption of noble gases on metallic surfaces. ${ }^{24}$ However, a high probability for elastic reflection was found there only for light noble gases on a clean metallic surface, whereas the sticking coefficient on the gas-covered surface was close to 1 . As both $\mathrm{Na}$ and $\mathrm{Cl}$ are much lighter than $\mathrm{Xe}$, the same high probability for elastic reflection in our case seems only to be possible if a large number of $\mathrm{NaCl}$ molecules effectively take part in a collision with Xe so that the effective mass of the surface "molecule" acting as the collision partner of the impinging Xe atom is correspondingly large.

The desorption behavior, on the other hand, found in our study is well known from several experiments of noble-gas desorption where apparent zeroth-order desorption out of the first layer of adsorbed noble gases has been found. ${ }^{25,26}$ Zeroth-order desorption can occur if two adsorbed phases, $2 \mathrm{D}$ gas and $2 \mathrm{D}$ solid, are present simultaneously on the surface. Desorption is then explained by the mixing of at least two desorption channels. One involves melting of the ordered islands and desorption out of the lattice gas, the other involves desorption from the second layer on top of the ordered islands.

$\mathrm{Xe}$ adsorption on the $\mathrm{Ge}(100)$ surface was found to be completely different from the results obtained on the $\mathrm{NaCl}$ surface. Obviously, no hexagonal or nearly hexagonal structures could be formed due to the strong corru- gation of the $\mathrm{Ge}(100)$ surface. These adsorbed phases have to be ordered and must be adsorbed on defined sites. This is indicated by the changes in the I-V curves of half order as well as of integral order spots after the jumps in the spot intensity. In contrast, a completely disordered adsorption would only lead to damping of the spot intensities without changing the I-V curves qualitatively. Since no additional superstructure spots could be observed Xe must form $(2 \times 1)$ structures on the surface with the occupation of nearest-neighbor (NN) substrate sites. As the natural Xe-Xe spacing in the bulk is about $10 \%$ larger than the Ge NN distance the Xe layers have to be compressed by this amount in one direction. A very similar behavior was found during Xe adsorption on the $\mathrm{Ge}(111)$ surface by Packard and $\mathrm{Webb}^{23}$ where again only jumps in intensity of substrate spots were observed during Xe adsorption. These authors carried out model calculations and found that this compression is energetically feasible on $\mathrm{Ge}(111)$. This finding can be transferred to $\mathrm{Ge}(100)$ if the assumption of a similar corrugation of the substrate potential surface in $\mathrm{Ge}(100)$ as on $\mathrm{Ge}(111)$ can be made. This kind of calculation still has to be carried out.

\section{CONCLUSIONS}

Xenon on $\mathrm{NaCl}(100)$ shows two well-ordered adsorbed phases which can be easily interpreted as a $2 \mathrm{D}$ and a $3 \mathrm{D}$ phase. The structure of the $2 \mathrm{D}$ phase is nearly hexagonal and is commensurate with the substrate only along one direction but incommensurate along the other direction. The heat of transition at the gas-2D phase transition is in good agreement with previous results obtained by Hardy et al. ${ }^{21}$ and is only slightly larger than the bulk condensation enthalpy indicating that the magnitude of adsorbate-substrate and adsorbate-adsorbate interactions is very similar in this system.

On Ge(100), on the other hand, Xe adsorbs in commensurate structures. The large corrugation of the $\mathrm{Ge}(100)$ surface enables a uniaxial compression of the Xe layer of about $10 \%$. Heats of transition are larger than those obtained on $\mathrm{NaCl}(100)$ but smaller than those found on metal surfaces.

\section{ACKNOWLEDGMENT}

This work was supported by the Deutsche Forschungsgemeinschaft through Project No. Pf238/1.

\footnotetext{
${ }^{1}$ H. Freimuth, H. Wiechert, H.P. Schildberg, and H.J. Lauter, Phys. Rev. B 42, 587 (1990), and references cited therein.

${ }^{2}$ K. Kern and G. Comsa, in Chemistry and Physics of Solid Surfaces VII, edited by R. Vanselow and R. Howe, Springer Series in Surface Science Vol. 10 (Springer, Berlin, 1988), p. 65.

${ }^{3}$ J. Cui and S.C. Fain, Phys. Rev. B 39, 8628 (1989).
}

\footnotetext{
${ }^{4}$ R. Marx, Phys. Rep. 125, 1 (1985).

${ }^{5} \mathrm{M}$. den Nijs, in Phase Transitions and Critical Phenomena, edited by C. Domb and J.L. Lebowitz (Academic Press, New York, 1988), Vol. 12.

${ }^{6}$ A. Glachant and U. Bardi, Surf. Sci. 87, 87 (1979); M.A. Chesters and J. Pritchard, ibid. 28, 460 (1971).

${ }^{7}$ P.W. Palmberg, Surf. Sci. 25, 598 (1971).

${ }^{8}$ T. Meichel, J. Suzanne, C. Girard, and C. Girardet, Phys.
} 
Rev. B 38, 3781 (1988).

${ }^{9}$ S. Fölsch, U. Barjenbruch, and M. Henzler, Thin Solid Films 172, 123 (1989).

${ }^{10}$ A. Klekamp and E. Umbach, Surf. Sci. 284, 291 (1993).

${ }^{11}$ R. Rossmann, H.L. Meyerheim, V. Jahns, J. Wever, W. Moritz, D. Wolf, D. Dornisch, and H. Schulz, Surf. Sci. 279, 199 (1992).

${ }^{12}$ M. Henzler, M. Horn v. Hoegen, and U. Köhler, in Festkörperprobleme (Advances in Solid State Physics), edited by P. Grosse (Pergamon, Braunschweig, 1992), Vol. 32, p. 333.

${ }^{13}$ J.A. Venables, G.D.T. Spiller, and M. Hanbücken, Rep. Prog. Phys. 47, 399 (1984).

${ }^{14}$ C. Schwennicke, J. Schimmelpfennig, and H. Pfnür, Surf. Sci. 293, 57 (1993).

${ }^{15}$ H. Pfnür, C. Schwennicke, and J. Schimmelpfennig, in Adsorption on Ordered Surfaces of Ionic Solids and Thin Films, edited by H.J. Freund and E. Umbach, Springer Series in Surface Science (Springer, Berlin, 1993).

${ }^{16}$ J. Heidberg, E. Kampshoff, O. Schönekäs, H. Stein, and H.
Weiss, Ber. Bunsenges. Phys. Chem. 94, 127 (1990).

${ }^{17} \mathrm{~J}$. Schimmelpfennig, S. Fölsch, and M. Henzler, Surf. Sci. 250, 198 (1991).

${ }^{18}$ U. Scheithauer, G. Meyer, and M. Henzler, Surf. Sci. 178, 441 (1986).

${ }^{19}$ S.D. Kevan, Phys. Rev. B 32, 2344 (1985).

${ }^{20}$ G. Pollack, Rev. Mod. Phys. 36, 748 (1964).

${ }^{21}$ J.P. Hardy, G.E. Ewing, R. Stables, and C.J.S.M. Simpson, Surf. Sci. 159, L474 (1985).

${ }^{22}$ C.W. Leming and G.L. Pollack, Phys. Rev. B 2, 3323 (1970).

${ }^{23}$ W.E. Packard and M.B. Webb, Surf. Sci. 195, 371 (1988).

${ }^{24} \mathrm{H}$. Schlichting, D. Menzel, T. Brunner, and W. Brenig, J. Chem. Phys. 97, 4453 (1992).

${ }^{25}$ H. Schlichting, Ph.D. thesis, Technische Universität München, 1990; H. Schlichting and D. Menzel (unpublished).

${ }^{26}$ J.A. Venables and M. Bienfait, Surf. Sci. 61, 667 (1976); Surf. Sci. 64, 425 (1977). 
(a)

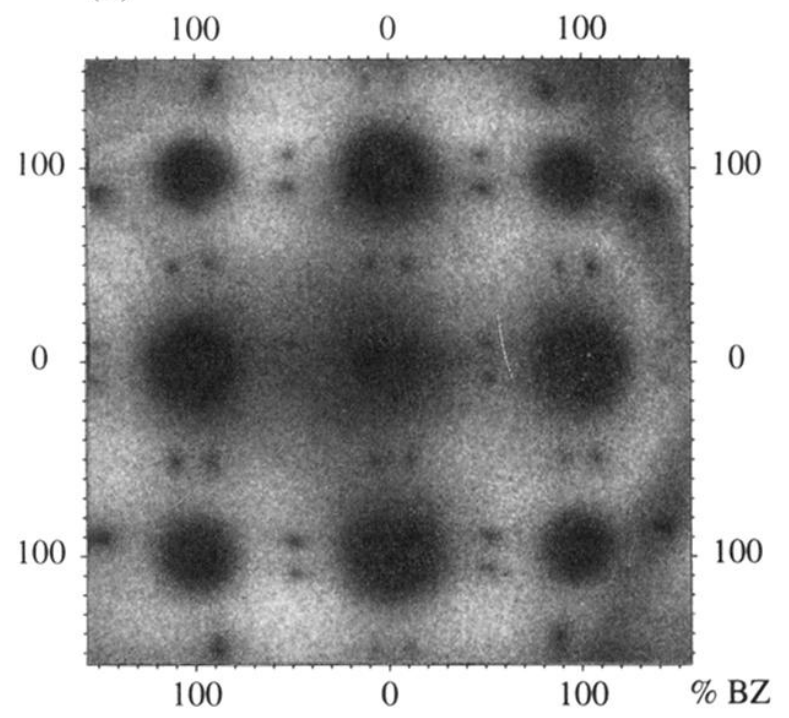

(b)

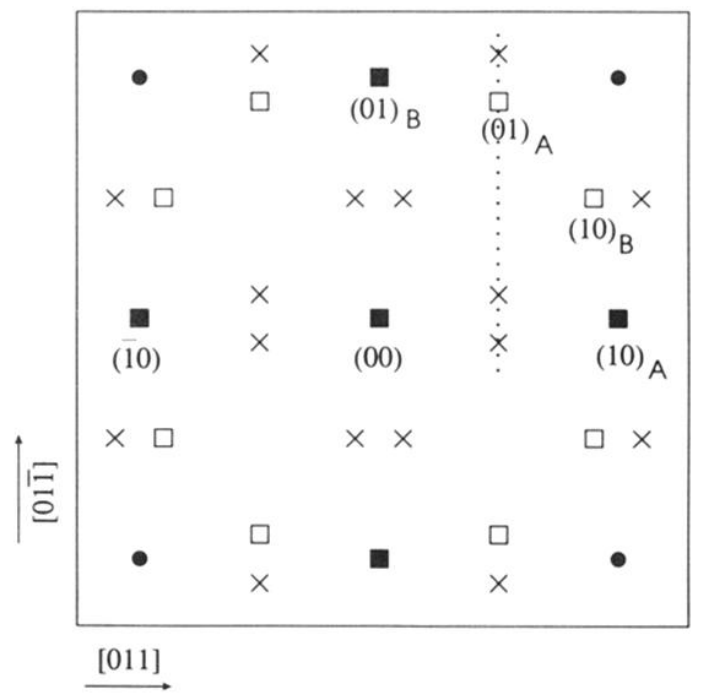

FIG. 1. (a) LEED pattern of $\mathrm{Xe}$ adsorbed on $\mathrm{NaCl}$ at $T=50 \mathrm{~K}, p=1 \times 10^{-5} \mathrm{~Pa}$, and $E=82 \mathrm{eV}$ showing the $(00)$ spot in the middle. Dark spots are the $\mathrm{NaCl}$ spots. Their width is due to overexposure and is not real. Distances between diffraction spots are measured in units of the separation between (00) and (10) spots of $\mathrm{NaCl}$, corresponding to the diameter of the first Brillouin zone (BZ). (b) Schematic of the LEED pattern shown in (a). Open squares, single diffraction adsorbate spots; filled squares, spots common to adsorbate and substrate; filled circles, substrate spots; crosses, multiplescattering spots; dots, path of scan in Fig. 2. 


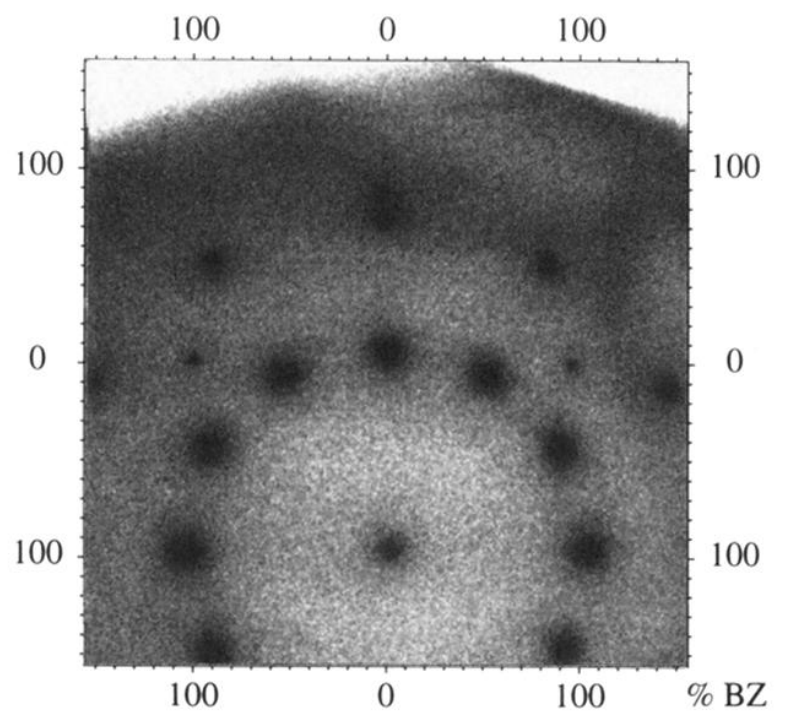

FIG. 4. LEED pattern of the 3D bulk structure of Xe condensed on $\mathrm{NaCl}$. Note that the (00) spot is at position $(0$, $-90)$. 